\title{
Urinary low-molecular-weight protein excretion in pediatric idiopathic nephrotic syndrome
}

\author{
Hassib Chehade • Paloma Parvex • Antoine Poncet • \\ Dominique Werner • Dolores Mosig • Francois Cachat • \\ Eric Girardin
}

Received: 9 March 2013 / Revised: 28 May 2013 / Accepted: 1 July 2013 /Published online: 15 August 2013

(C) IPNA 2013

\begin{abstract}
Background Minimal change disease (MCD) and focal segmental glomerulosclerosis (FSGS) are the most common causes of idiopathic nephrotic syndrome (INS). We have evaluated the reliability of urinary neutrophil-gelatinaseassociated lipocalin (uNGAL), urinary alpha1-microglobulin (u $\alpha 1 \mathrm{M}$ ) and urinary $N$-acetyl-beta-D-glucosaminidase (ußNAG) as markers for differentiating MCD from FSGS. We have also evaluated whether these proteins are associated to INS relapses or to glomerular filtration rate (GFR).
\end{abstract}

H. Chehade $(\bowtie) \cdot$ D. Mosig $\cdot$ F. Cachat $\cdot$ E. Girardin Division of Pediatric Nephrology, Department of Pediatrics, Lausanne University Hospital, Rue Bugnon 46, 1011 Lausanne, Switzerland

e-mail: hassib.chehade@chuv.ch

D. Mosig

e-mail: dolores.mosig@chuv.ch

F. Cachat

e-mail: francois.cachat@chuv.ch

E. Girardin

e-mail: eric.girardin@chuv.ch

P. Parvex

Division of Pediatric Nephrology, Department of Pediatrics, Geneva University Hospital, Rue Willy-Donzé 6, 1205 Geneva, Switzerland

e-mail: paloma.parvex@hcuge.ch

A. Poncet

CRC \& Division of Clinical-Epidemiology, Department of Health and Community Medicine, University of Geneva \& University Hospitals of Geneva, Rue Willy-Donzé 6, 1205 Geneva, Switzerland

e-mail: antoine.poncet@hcuge.ch

D. Werner

Central Chemistry Laboratory, Lausanne University Hospital, Rue Bugnon 46, 1011 Lausanne, Switzerland

e-mail: dominique.werner@chuv.ch
Methods The patient cohort comprised 35 children with MCD and nine with FSGS; 19 healthy age-matched children were included in the study as controls. Of the 35 patients, 28 were in remission (21 MCD, 7 FSGS) and 16 were in relapse (14 MCD, 2 FSGS). The prognostic accuracies of these proteins were assessed by receiver operating characteristic (ROC) curve analyses.

Results The level of uNGAL, indexed or not to urinary creatinine (uCreat), was significantly different between children with INS and healthy children $(p=0.02)$, between healthy children and those with FSGS $(p=0.007)$ and between children with MCD and those with FSGS $(p=0.01)$. It was not significantly correlated to proteinuria or GFR levels. The ROC curve analysis showed that a cut-off value of $17 \mathrm{ng} / \mathrm{mg}$ for the uNGAL/uCreat ratio could be used to distinguish MCD from FSGS with a sensitivity of 0.77 and specificity of 0.78 . u $\beta \mathrm{NAG}$ was not significantly different in patients with MCD and those with FSGS $(p=0.86)$. Only $\mathrm{u} \alpha 1 \mathrm{M}$, indexed or not to uCreat, was significantly $(p<0.001)$ higher for patients in relapse compared to those in remission. Conclusions Our results indicate that in our patient cohort uNGAL was a reliable biomarker for differentiating MCD from FSGS independently of proteinuria or GFR levels.

Keywords Urinary NGAL $\cdot$ Low-molecular-weight proteins $\cdot$ Idiopathic nephrotic syndrome $\cdot$ Minimal change disease $\cdot$ Focal segmental glomerulosclerosis

\section{Introduction}

Idiopathic nephrotic syndrome (INS) remains the most frequent glomerular disease in children [1] with reported incidences of two to seven cases per 100,000 children [2]. It is caused by various entities that differ in their histopathological forms and clinical course [3]. Minimal change disease (MCD) and focal segmental glomerulosclerosis (FSGS) are 
the most common causes of INS, representing 80 and $20 \%$ of the cases, respectively [3]. The pathogenesis of this disease is still unknown, and its clinical outcomes are determined by their histopathological diagnosis and responsiveness to steroid treatment. Steroid-sensitive INS (SSINS) is due to MCD in $80 \%$ of cases and has a favorable long-term prognosis [3], while steroid-resistant INS (SRINS) is mostly due to FSGS and is significantly associated with poor outcomes $[4,5]$. INS can be associated with various proximal tubular dysfunctions, such as glycosuria, aminoaciduria and bicarbonate losses [6]. The results from a few studies have suggested that some low-molecular-weight proteins (LMWPs), such as urinary beta2-microglobulinuria $(\beta 2 \mathrm{M})$ $[6,7]$ or urinary retinol binding protein (RBP) [6], can predict steroid treatment responsiveness in INS. However, other studies have failed to prove a significant difference in the urinary excretion of $\beta 2 \mathrm{M}$ between SRINS and SSNS patients [8]. More recently, Bennett et al. [1] reported that urinary neutrophil gelatinase-associated lipocalin (uNGAL), which is a small protein belonging to the lipocalin superfamily, can be used to distinguish SSINS from SRINS. However, the literature regarding the use of this novel urinary marker in INS is limited to this one publication [1], and to date no studies evaluating the role of UNGAL in MCD and FSGS patients have been published.

The primary aim of our study was to evaluate the role of three urinary LMWPs, namely, uNGAL, urinary alpha 1microglobulin $(\mathrm{u} \alpha 1 \mathrm{M})$ and urinary $N$-Acetyl-beta-Dglucosaminidase ( $\mathrm{u} \beta \mathrm{NAG}$ ) in differentiating MCD from FSGS and to discuss the underlying pathophysiological mechanisms. A second aim was to evaluate whether these renal tubular proteinuria can be associated to INS relapses or to INS glomerular filtration rate (GFR) levels.

\section{Materials and methods}

\section{Study population}

This is a prospective cross-sectional study which received the approval of the local research ethics board; informed consent was obtained from all participants and/or their legal guardians. Forty-seven patients aged between 2 and 18 years, all diagnosed with INS, and 19 healthy age-matched controls, were included. Renal biopsy was performed in 44 patients with INS at the time of diagnosis for SRINS or steroid-dependent INS, or after three or more relapses in 1 year for frequent relapsers. Of the 47 patients with INS, three did not have a renal biopsy and were excluded from our study. The final study population $(n=44)$ were divided into three groups, with group 1 ( $n=35,35$ biopsied) including patients with MCD, group 2 ( $n=9,9$ biopsied) including patients with FSGS and group $3(n=19)$ including healthy control children. The median time with the interquartile range (IQR; 25th and 75th percentile) between initial diagnosis and time of inclusion in the study was $4[2 ; 6]$ and $4[3 ; 6]$ years for patients diagnosed with FSGS and MCD, respectively. Furthermore, children with INS were divided into two groups based on the urine specimen results independently of the histopathological diagnosis: (1) patients in remission (total $n$ of patients in remission 28: $21 \mathrm{MCD}, 7$ FSGS) and (2) patients in relapse (total $n$ of patients in relapse 16: 14 MCD, 2 FSGS). Remission was defined as a urinary protein over creatinine ratio of $\leq 20 \mathrm{~g} / \mathrm{mol}$ or $0.2 \mathrm{~g} / \mathrm{g}$. Relapse was defined as a measurement of $\geq 2+$ on urinary dipsticks for at least 3 consecutive days, as confirmed by a urine protein/creatinine ratio of $>200 \mathrm{~g} / \mathrm{mol}$ or $2 \mathrm{~g} / \mathrm{g}$. Urinary excretion of $\alpha 1 \mathrm{M}, \beta \mathrm{NAG}$ and NGAL were measured in all groups. The INS patients' demographic characteristics and treatment are summarized in Table 1.

Analytical methods

Blood samples with lithium heparinate were processed immediately for plasma creatinine determination, and spot urine samples were analyzed immediately for creatinine, uNGAL, $\mathrm{u} \alpha 1 \mathrm{M}$ and $\mathrm{u} \beta \mathrm{NAG}$ determination. Serum creatinine (SCreat) was measured on a Modular P system (Roche Diagnostics, Indianapolis, IN) using the kinetic colorimetric compensated Jaffe method from Roche Diagnostics. Inter-assay coefficients of variation obtained with the internal quality controls were $3.9 \%$ at $46 \mu \mathrm{mol} / 1$ and $2.4 \%$ at $108 \mu \mathrm{mol} / 1$. Urine creatinine (uCreat) was determined using the same method but was calibrated in on a homemade aqueous creatinine solution of $15.1 \mathrm{mmol} / \mathrm{l}$. Inter-assay CVs were $1.7 \%$ at $7.2 \mathrm{mmol} / \mathrm{l}$ and $0.7 \%$ at $12.8 \mathrm{mmol} / \mathrm{l}$. u $\beta \mathrm{NAG}$ was measured with a colorimetric assay using sodium-3-cresolsulfonphthaleinyl- $N$ acetyl- $\beta$-D-glucosaminide as substrate (Roche Applied Science, Rotkreuz, Switzerland) adapted on a Modular P and calibrated with the ußNAG standard from Roche Applied Science. Inter-assay CVs were $1.2 \%$ at $9 \mathrm{U} / 1$ and $0.9 \%$ at $90 \mathrm{U} / \mathrm{l}$. uNGAL was determined by a chemiluminescent microparticule immunoassay on an Architect i1000SR (Abbott Diagnostics, North Chicago, IL). During the entire study, inter-assay CVs determined on the three levels of internal quality controls were $\leq 2.2 \%$ (respective means of controls: 21,184 and $1,196 \mu \mathrm{g} / \mathrm{l}) . \mathrm{u} \alpha 1 \mathrm{M}$ was measured by immunonephelometry on a BN ProSpec analyzer (Siemens Healthcare Diagnostics, Tarrytown, NY) with an inter-assay CV of $5.3 \%$ at $37.7 \mathrm{mg} / \mathrm{l}$.

GFR estimation methods

The estimated GFR (eGFR) was calculated using the revised Schwartz and the new Quadratic formulas as follows: 
Table 1 Descriptive demographics

\begin{tabular}{|c|c|c|c|c|c|c|c|}
\hline $\mathrm{n}$ & 44 & MCD & FSGS & $p$ value $^{\mathrm{a}}$ & $\begin{array}{l}\text { Children in } \\
\text { relapse } \\
13\end{array}$ & $\begin{array}{l}\text { Children in } \\
\text { remission } \\
31\end{array}$ & $p$ value $^{\mathrm{a}}$ \\
\hline Median age (year) & $10[5 ; 13]$ & $10[5.2 ; 12]$ & $13[5 ; 16]$ & 0.41 & $9[4.7 ; 11.5]$ & $10[5.5 ; 13]$ & 0.32 \\
\hline Median weight (Kg) & $38.7[29.1 ; 56.5]$ & $36[30 ; 53]$ & $51[29 ; 69.7]$ & 0.58 & $33.5[23.3 ; 59.2]$ & $40.5[31 ; 53.2]$ & 0.68 \\
\hline Median height (cm) & $145[126 ; 159]$ & $145[131 ; 159]$ & $152[122 ; 159]$ & 0.87 & $138[120 ; 161]$ & $147[134 ; 158]$ & 0.47 \\
\hline Median uNGAL $(\mu \mathrm{g} / \mathrm{l})$ & $4.6[1.98 ; 12]$ & $2.7[1.9 ; 8.3]$ & $12.1[7.1 ; 45.4]$ & 0.01 & $3.5[1.7 ; 9.4]$ & $4.6[2.1 ; 12.0]$ & 0.68 \\
\hline Median ußNAG (U/l) & $5.9[4 ; 8.93]$ & $5.9[4.0 ; 10.2]$ & $5.9[4.0 ; 8.1]$ & 0.86 & $11.5[7.3 ; 18.9]$ & $4.0[4.0 ; 5.9]$ & 0.001 \\
\hline Median ua1M (mg/l) & $2[0.88 ; 4.72]$ & $1.7[0.8 ; 5.0]$ & $2.6[1.6 ; 4.1]$ & 0.35 & $6.8[2.5 ; 12.7]$ & $1.6[0.8 ; 2.6]$ & 0.002 \\
\hline Median uNGAL/uCreat $(\mu \mathrm{g} / \mathrm{mmol})$ & $1.24[0.58 ; 5.29]$ & $1.0[0.5 ; 1.8]$ & $8.3[2.1 ; 27.4]$ & 0.02 & $1.5[0.6 ; 1.9]$ & $1.1[0.6 ; 7.2]$ & 0.76 \\
\hline Median ußNAG/uCreat (U/mmol) & $2.24[1.18 ; 6.8]$ & $2.1[1.1 ; 5.9]$ & $4.8[1.4 ; 9.9]$ & 0.38 & $3.1[1.7 ; 6.8]$ & $1.8[0.9 ; 5.7]$ & 0.31 \\
\hline Median ua1M /uCreat (mg/mmol) & $0.3[0.2 ; 0.73]$ & $0.3[0.2 ; 0.8]$ & $0.3[0.3 ; 0.6]$ & 0.68 & $1.6[0.5 ; 2.9]$ & $0.2[0.2 ; 0.3]$ & 0.001 \\
\hline Median eGFR using Schwartz formula & $112[94 ; 134]$ & $113[95 ; 138]$ & $109[80 ; 124]$ & 0.20 & $114[94 ; 140]$ & $111[94 ; 129]$ & 0.69 \\
\hline Median eGFR using Quadratic formula & $108[98 ; 116]$ & $108[98 ; 116]$ & $106[98 ; 111]$ & 0.56 & $108[98 ; 117]$ & $109[98 ; 114]$ & 0.75 \\
\hline $\begin{array}{l}\text { Number of children under steroid } \\
\text { treatment }\end{array}$ & 31 & 26 & 5 & - & 9 & 22 & - \\
\hline $\begin{array}{l}\text { Number of children under cyclosporine } \\
\text { treatment }\end{array}$ & 13 & 6 & 7 & - & 3 & 10 & - \\
\hline $\begin{array}{l}\text { Number of children under CellCept } \\
\text { treatment }\end{array}$ & 12 & 8 & 4 & - & 1 & 11 & - \\
\hline
\end{tabular}

$\mathrm{MCD}$, minimal change disease; FSGS, focal segmental glomerulosclerosis; uNGAL, urinary Neutrophil-gelatinase-associated lipocalin; $\mathrm{u} \beta \mathrm{NAG}$, urinary $N$-Acetyl-Beta-D-glucosaminidase; u $\alpha 1 \mathrm{M}$, urinary alpha1-microglobulin; uCreat, urinary creatinine; eGFR, estimated glomerular filtration rate expressed in $\mathrm{ml} / \mathrm{mn}$ per $1.73 \mathrm{~m}^{2}$. Continuous parameters are presented as the median with the interquartile range (IQR; $25 \% ; 75 \%$ as lower and upper quartiles) given in square brackets. ${ }^{a} p$ values were obtained using the Wilcoxon-Mann-Whitney test.

- Revised Schwartz formula [9]:

$$
\text { eGFR }=0.413 \times(\text { Height } / \text { SCreat })
$$

- Quadratic formula [10]:

- Males:

$$
\begin{aligned}
\text { eGFR }= & 0.68 \times(\text { Height } / \text { SCreat })-0.0008 \\
& \times(\text { Height } / \text { SCreat })^{2}+0.48 \times \text { age }-21.53
\end{aligned}
$$

- Females:

$$
\begin{aligned}
\mathrm{eGFR}= & 0.68 \times(\text { Height } / \text { SCreat })-0.0008 \\
& \times(\text { Height } / \text { SCreat })^{2}+0.48 \times \text { age }-25.68
\end{aligned}
$$

In all formulas, height is in centimeters, SCreat is in milligrams per deciliter, eGFR is in milliliter per minute per $1.73 \mathrm{~m}^{2}$

Histological evaluation of the renal biopsies

Renal tissue was obtained by percutaneous biopsy in 44 children diagnosed with INS. All specimens were fixed in Bouin's alcoholic solution, post-fixed in formalin and then embedded in Paraffin. Sections were cut at a thickness of
2-3 $\mu \mathrm{m}$ and stained routinely with hematoxylin and eosin, periodic acid-Schiff and periodic acid-silver-methenamine. All biopsy sections were examined by electron microscopy.

\section{Statistical analysis}

Collected data were analyzed using R software, version 2.13.1 (R Foundation for Statistical Computing, Vienna, Austria; www.R-project.org). Data were reported as medians with upper and lower quartiles for continuous parameters (IQR; 25th; 75th percentile), and categorical variables are expressed as percentages. The bilateral Fisher exact test was used for the comparison of nominal variables. For continuous variables, the difference was computed with the Mann-Whitney $U$ test or the $t$ test according to the number and normality of the data. Thus, for the comparisons between MCD and FSGS groups we used the Mann-Whitney $U$ test, whereas the $t$ test was used to compare the log-transformed uNGAL/uCreat ratio values between the control group and the pooled MCD and FSGS groups. Prognostic accuracies were assessed by receiver operating characteristic (ROC) curve analyses providing the area under the curve (AUC) and $95 \%$ confidence intervals using a nonparametric approach as proposed by Delong [11]. Finally, correlation analysis of $\mathrm{u} \alpha 1 \mathrm{M}, \mathrm{u} \beta \mathrm{NAG}$ and $\mathrm{uNGAL}$ with the urinary protein excretion indexed to urinary creatinine was assessed using the Spearman rank correlation test, and linear regression analysis was performed using the logarithmic value of these 
three parameters to support visual inspections. Statistical significance was assessed at the 0.05 level for all analyses.

\section{Results}

\section{uNGAL excretion in INS}

\section{uNGAL excretion in children with MCD and FSGS}

The median [IQR] uNGAL concentration was significantly $(p=0.01)$ higher in patients with FSGS than in patients with MCD (12.1 [7.1; 45.4] vs. $2.7[1.9 ; 8.3] \mu \mathrm{g} / 1$, respectively). The results remained unchanged when uNGAL was indexed to uCreat $(8.3[2.1 ; 27.4]$ vs. $1.0[0.5 ; 1.8] \mu \mathrm{g} / \mathrm{mmol}$, respectively; $p=0.02$ ). In addition, uNGAL, indexed or not to uCreat, was also significantly different between INS and healthy children $(p=0.02)$ and between healthy and children with FSGS $(p=0.007)$. The AUC for the uNGAL/uCreat ratio as a biomarker to distinguish FSGS from MCD was 0.76
$[0.57 ; 0.95](p=0.01)$ and showed an optimized sensitivity of 0.77 and specificity of 0.78 with an optimal cut-off value, calculated according to Youden et al. [12], of $1.93 \mu \mathrm{g} / \mathrm{mmol}$ (corresponding to $17 \mathrm{ng} / \mathrm{mg}$ ) (Fig. 1a).

\section{Correlation between uNGAL excretion and proteinuria level}

The correlation between UNGAL indexed or not to the $\mathrm{uCreat}$ and the protein excretion related to uCreat in the whole study group with INS was close to 0 (Spearman correlation coefficient $r-0.05$ and -0.04 with $p$ values of 0.75 and 0.81 , respectively) (Fig. 2a) and was also observed in MCD and FSGS patients. This latter observation demonstrated that the higher uNGAL values in FSGS, which enabled this group to be distinguished from the MCD group, were not related to a higher urinary protein/creatinine ratio. There was also no significant difference in median uNGAL indexed to the uCreat between patients in relapse versus those in remission ( $p=0.76$ ) (Fig. 3a).
Fig. 1 Receiver operator characteristic curves (ROC) for urinary neutrophil gelatinaseassociated lipocalin $(u N G A L)$ (a), urinary alpha 1microglobulin $(u \alpha 1 M)(\mathbf{b})$ and urinary $N$-acetyl-beta-Dglucosaminidase $(u \beta N A G)(\mathbf{c})$ for differentiating minimal change disease (MCD) from focal segmental glomerulosclerosis (FSGS). uNGAL was found to have significant power to distinguish MCD from FSGS with an area under the curve (AUC) of 0.76 (interquartile range $[0.57 ; 0.95]$ ) $(p=0.01)$ a
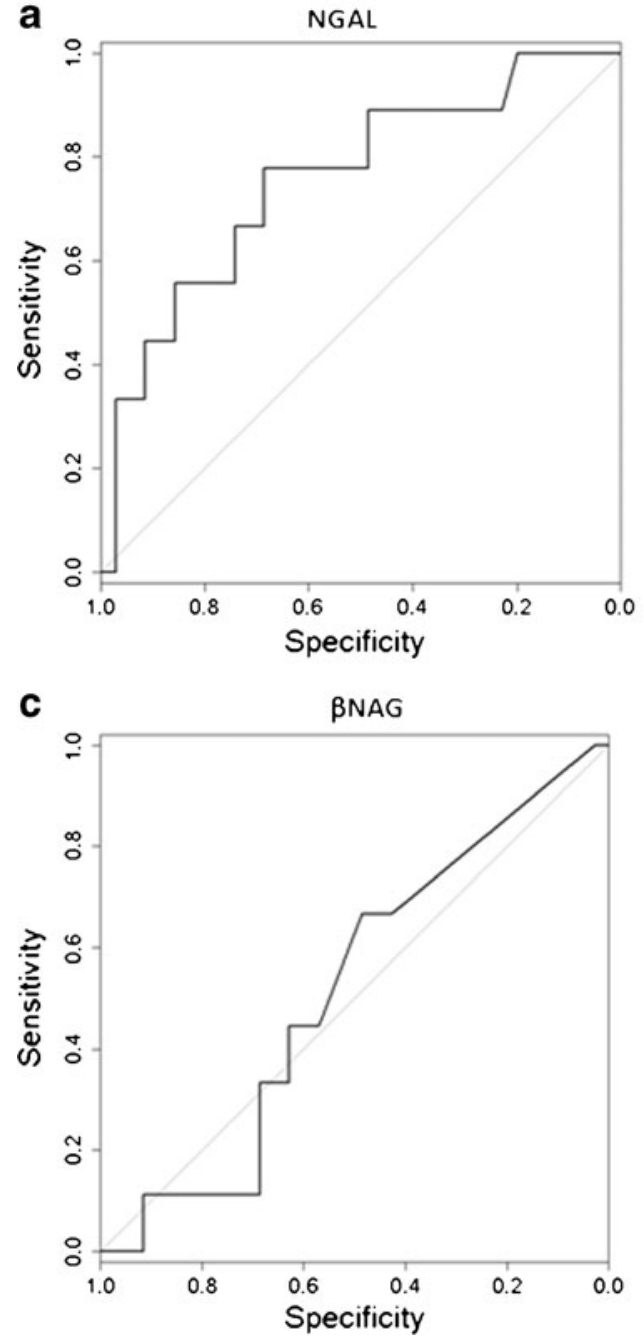

b

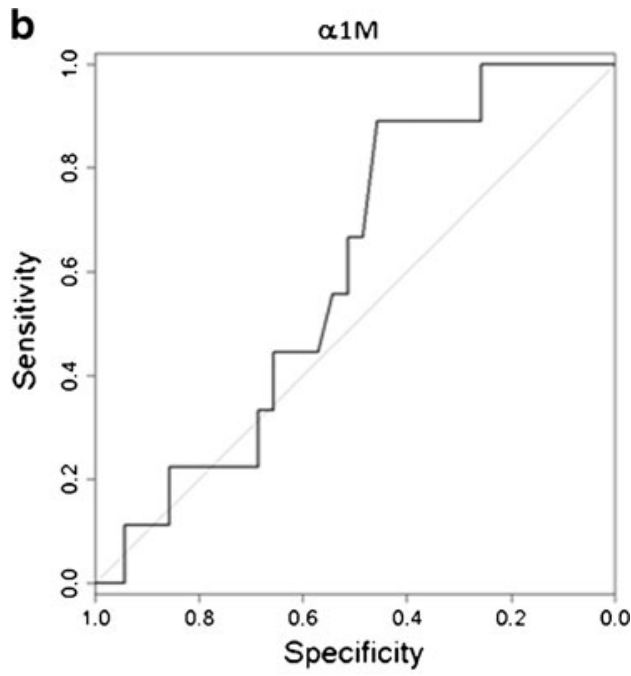




\section{Correlation between $u N G A L$ excretion and eGFR}

For the each patient group, there was no significant correlation between UNGAL and the UNGAL indexed to the uCreat and the eGFR ( $p=0.26$ and 0.45 , respectively, with the revised Schwartz formula, and $p=0.57$ and 0.48 , respectively, with the new Quadratic formula). Sub-analysis for the FSGS and MCD groups also showed no significant correlation between eGFR and $\mathrm{UNGAL}$ excretion. In addition, using a multivariate analysis in which eGFR was a controlling co-variate, we found that UNGAL distinguished between FSGS and MCD and that this difference was independent of GFR levels. These results indicated that the highest UNGAL levels obtained in our FSGS patients were not related to renal disease severity.

$\mathrm{u} \alpha 1 \mathrm{M}$ excretion in INS

\section{uQ1M excretion in children with MCD and FSGS}

The median [IQR] $\mathrm{u} \alpha 1 \mathrm{M}$ concentration in FSGS patients was not significantly different $(p=0.35)$ from that in MCD patients $(2.6[1.6 ; 4.1]$ vs. $1.7[0.8 ; 5] \mathrm{mg} / \mathrm{l}$, respectively). $\mathrm{u} \alpha 1 \mathrm{M}$ indexed to $\mathrm{uCreat}$ was also not significantly different between FSGS and MCD patients $(0.3[0.3 ; 0.6]$ vs. $0.3[0.2$;
$0.8] \mathrm{mg} / \mathrm{mmol}$, respectively). The AUC for the $\mathrm{u} \alpha 1 \mathrm{M}$ as a tool to distinguish MCD from FSGS was $0.52[0.32 ; 0.71]$ (Fig. 1b); this value was not significant $(p=0.86)$. These results remained unchanged when $\mathrm{u} \alpha 1 \mathrm{M}$ was indexed to uCreat (AUC 0.60 [0.37; 0.82]; not significant $p=0.38$ ).

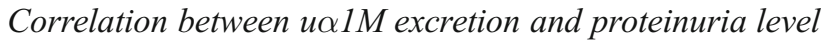

For the whole group with INS, the correlation between the $\mathrm{u} \alpha 1 \mathrm{M}$ related to the $\mathrm{uCreat}$ and the protein excretion corrected for uCreat showed a positive and significant correlation coefficient ( $r=0.79, p<0.001)$ (Fig. 2b). In addition, median $\mathrm{u} \alpha 1 \mathrm{M}$ related or not to the $\mathrm{uCreat}$ for patients in relapse was significantly higher than that for those children in remission ( $p=0.001$ ) (Fig. 2c), independently of the histopathological diagnosis.

\section{Correlation between $u \alpha 1 M$ excretion and eGFR}

The correlation between the $\mathrm{u} \beta \mathrm{NAG}$ and the eGFR using the revised Schwartz and the new Quadratic formulas was poor and not significant $(r=0.28$ and $0.24, p=0.067$ and 0.129 , respectively), with no differences between the FSGS and MCD groups.
Fig. 2 Scattergraph depicting the relationships of the logarithms of urinary neutrophil gelatinase-associated lipocalin (uNGAL) to urinary creatinine (uCreat) ratio (a), $\mathrm{u} \alpha 1 \mathrm{M}$ to $\mathrm{uCreat}$ ratio (b) and $\mathrm{u} \beta \mathrm{NAG}$ to uCreat ratio (c) with the logarithms of the urinary protein (uProt) to uCreat (uProt/uCreat) ratio in minimal change disease (MCD) patients (dotted lines) and focal seymental glomerulosclerosis (FSGS) (dashed lines). The correlation coefficients are: $r=-0.05$, $p=0.75$ (uNGAL), $r=0.79$, $p<0.001$ (u $\alpha 1 \mathrm{M})$ and $r=0.12$, $p=0.38$ (ußNAG) a

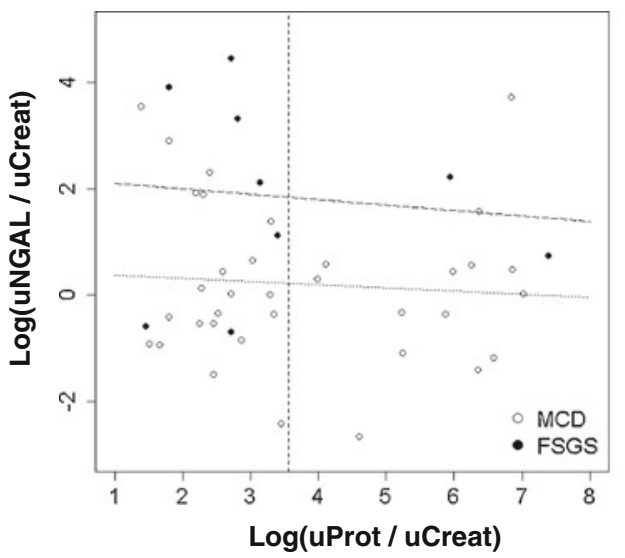

C

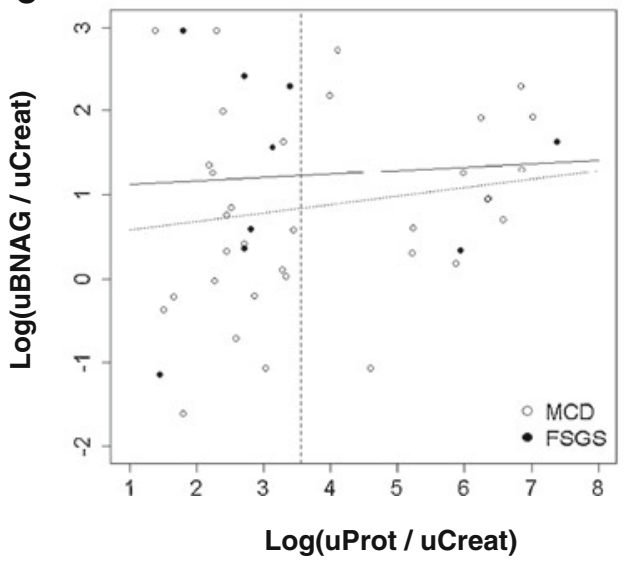

b

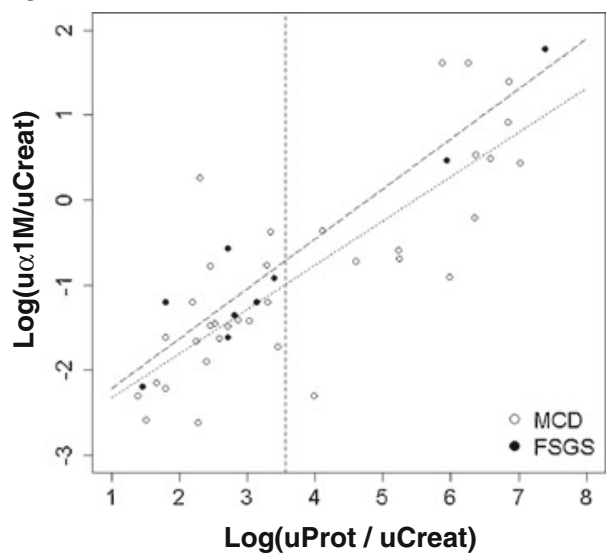


Fig. 3 Box plot for the median uNGAL to uCreat ratio (a), median $\mathrm{u} \alpha 1 \mathrm{M}$ to $\mathrm{uCreat}$ ratio (b) and median urinary $N$-Acetyl-betaD-glucosaminidase (u $\beta \mathrm{NAG}$ ) to urinary creatinine (uCreat) ratio (c) for differentiating idiopathic nephrotic syndrome (INS) patients in remission (RE) from INS patients in relapse (RL). The median $\mathrm{u} \alpha 1 \mathrm{M} / \mathrm{uCreat}$ ratio for patients in $\mathrm{RL}$ was significantly higher than that for those in $\mathrm{RE}(p<0.001)$
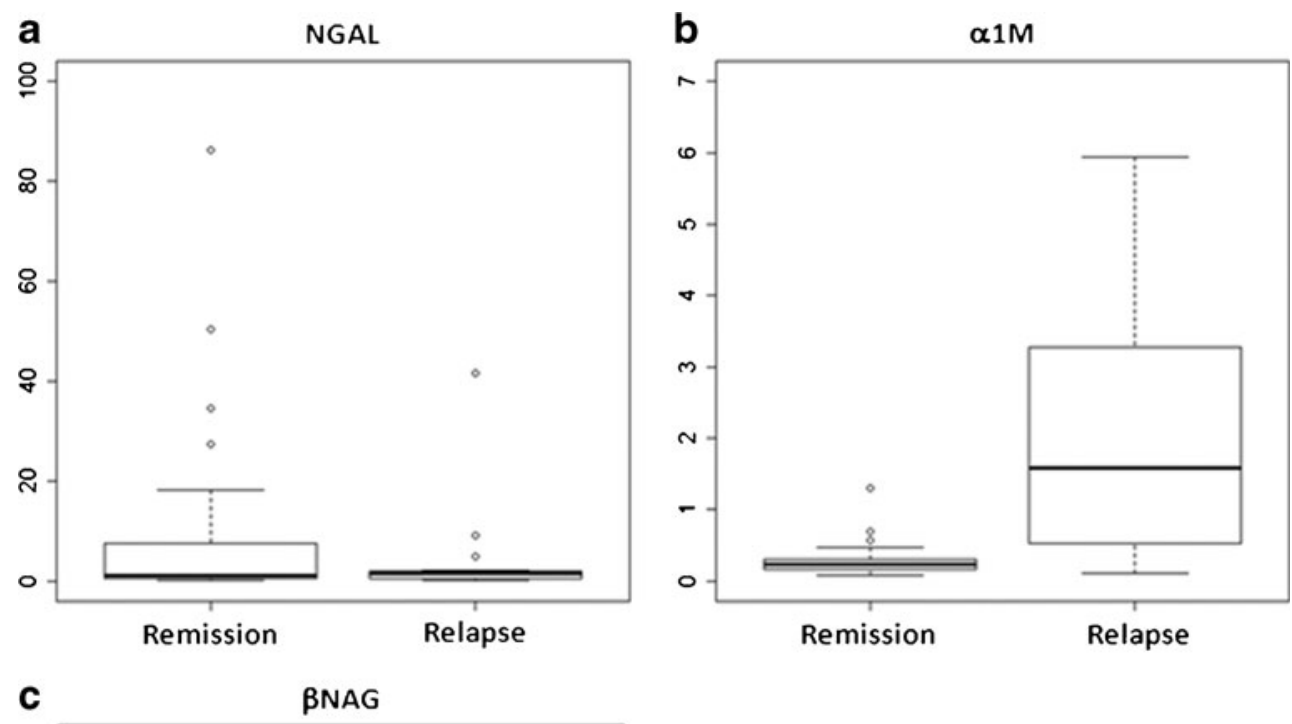

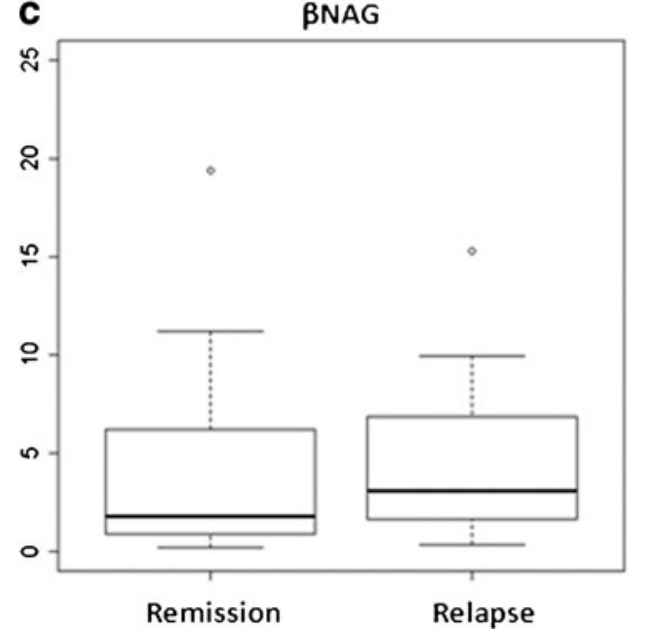

$\mathrm{u} \beta \mathrm{NAG}$ excretion in INS

\section{u $\beta N A G$ excretion in children with MCD and FSGS}

The median [IQR] u $\beta$ NAG concentration in FSGS patients was not significantly different $(p=0.86)$ from that in MCD patients $(5.9$ [4.0; 8.1] vs. 5.9 [4.0;10.2] U/1, respectively). $\mathrm{u} \beta \mathrm{NAG}$ indexed to uCreat also did not significantly differ between the FSGS and MCD groups (4.8 [1.4; 9.9] vs. 2.1 $[1.1 ; 5.9] \mathrm{U} / \mathrm{mmol}$, respectively). The AUC for $\mathrm{u} \beta \mathrm{NAG}$ to distinguish MCD from FSGS was 0.60 [0.42; 0.78] (Fig. 1c) (not significant $p=0.35$ ). These results remained unchanged when $\mathrm{u} \beta \mathrm{NAG}$ was indexed to uCreat (AUC 0.54 [0.34; 0.75 ]; not significant $p=0.68$ ).

\section{Correlation between $\mathrm{u} \beta N A G$ excretion and proteinuria level}

The correlation between $\mathrm{u} \beta \mathrm{NAG}$ and the protein excretion corrected to uCreat was very low and not significant ( $r=0.12, p=0.38$ ) (Fig. 2c). On the other hand, there was no significant difference in median $\mathrm{u} \beta \mathrm{NAG}$ indexed to the
$\mathrm{uCreat}$ between patients in relapse versus those in remission $(p=0.31)$ (Fig. 3c).

\section{Correlation between $u \beta N A G$ excretion and $e G F R$}

The correlation between the $\mathrm{u} \beta \mathrm{NAG}$ and the eGFR using the revised Schwartz and the new Quadratic formulas was poor and not significant $(r=0.25$ and $0.24 ; p=0.106$ and 0.120 , respectively), again with no differences between the FSGS and MCD groups.

\section{Discussion}

Several tubular urinary markers have been studied in INS with conflicting results. Sesso et al. [6] showed that urinary RBP and $\beta 2 \mathrm{M}$ levels may be helpful biomarkers for distinguishing established SRINS from SSINS. Caliskan et al. [8] and Valles et al. [13] showed no difference in the urinary $\beta 2 \mathrm{M}$ excretion in children who are more likely to be responsive to steroid treatments. During the past few years, 
major efforts have been made to identify new urinary biomarkers that would allow physicians to differentiate SRINS from SSINS. Woroneicki et al. evaluated various urinary proteomic markers [14] and urinary cytokine panels [15] to identify whether any of these factors could distinguish SRINS from SSINS, but the results were nonconclusive. Bennett et al. [1] recently demonstrated that the ratio of uNGAL to urinary creatinine could be used to differentiate SSNS from SRNS; in addition, this new urinary biomarker was correlated with disease severity in SRNS.

Our study cohort comprised children with biopsy-proven established kidney disease. In this cohort, our results showed that using a cut-off value of $17 \mathrm{ng} / \mathrm{mg}$, the uNGAL/uCreat ratio enabled MCD to be distinguished from FSGS with an AUC of $0.75(p=0.01)$ and an optimized sensitivity of 0.77 and specificity of 0.78 . This finding has important implications in clinical practice as we have demonstrated the role of uNGAL as a novel biomarker in children with INS, thereby confirming the findings of Bennett et al. [1]. We also found that UNGAL can readily distinguish between the two different histopathological glomerular diseases, independent of GFR or proteinuria levels.

The underlying pathophysiological mechanisms of uNGAL excretion/metabolism in INS are still unknown. Different hypotheses have been explored in previous investigations, such as an injured glomerular barrier, a possible adaptive tubular response to injury by NGAL inhibition of apoptosis or an abnormal cubulin-megalin transporter [16].

Regarding urinary LMWP excretion in children with INS in remission or in relapse, we obtained discordant results. There was a significant increase in the median $\mathrm{u} \alpha 1 \mathrm{M} / \mathrm{uCreat}$ ratio in children with relapse compared to those in remission. This increase in the $\mathrm{u} \alpha 1 \mathrm{M} / \mathrm{uCreat}$ ratio was significant and positively related to the level of proteinuria $(r=0.79)$. However, the medians of the uNGAL/uCreat and $u \beta N A G / u C r e a t$ ratios were not significantly different between children in remission and children in relapse. The observation of increasing $\mathrm{u} \alpha 1 \mathrm{M}$ concentration in relapsed INS compared to the remission state is consistent with the previously described hypothesis that proteinuria decreases the absorption of LWMP through competition for common transport mechanisms [17-20] but it also does not exclude the association of proximal tubular injury related to interstitial fibrosis from the underlying disease [21-23]. This proximal tubular injury could also be secondary to tubular ischemia, as reported by Sesso et al. [6]. There is therefore a need for studies on the measurement of cellular tubular oxygenation during remissions and relapses to confirm or not the Sesso hypothesis.

In brief, mechanisms of urinary excretion of LWMP in the condition of relapsed INS are still not well established and may be secondary to several tubular pathophysiological processes. A delicate and complicated interplay, as yet not completely resolved, between uNGAL, the glomerular filtration process; proximal tubular reabsorption and distal tubular secretion might explain the lack of difference between uNGAL concentration in children with INS in relapse and in remission.

There are a number of limitations to this study. First, the majority of children with FSGS were already on calcineurin inhibitor (CNI) treatment at the date of inclusion. Several studies showed contrasting results regarding the impact of CNI on uNGAL excretion in children with INS $[1,24]$. The number of children included was fairly small, especially in the FSGS group, and therefore, the impact of CNI could not be evaluated in our study group. Secondly, although we found a uNGAL cut-off with good sensitivity and specificity, additional larger studies are needed to validate this new urinary cut-off as a potential alternative marker that could replace renal biopsy. Finally, given the fact that all our FSGS patients had histological lesions not otherwise specified, we could not assess the role of FSGS subtypes on uNGAL excretion.

To our knowledge, this study is the first to demonstrate that UNGAL can distinguish children with biopsy-proven MCD from those with FSGS In addition, we provide a urinary NGAL to creatinine ratio cut-off point that can be helpful in clinical practice to differentiate these two histopathological diagnoses.

\section{References}

1. Bennett MR, Piyaphanee N, Czech K, Mitsnefes M, Devarajan P (2012) NGAL distinguishes steroid sensitivity in idiopathic nephrotic syndrome. Pediatr Nephrol 27:807-12

2. Lahdenkari AT, Suvanto M, Kajantie E, Koskimies O, Kestilä M, Jalanko H (2005) Clinical features and outcome of childhood minimal change nephrotic syndrome: is genetics involved? Pediatr Nephrol 20:1073-80

3. Eddy AA, Symons JM (2003) Nephrotic syndrome in childhood. Lancet 362:629-39

4. Cattran DC, Rao P (1998) Long-term outcome in children and adults with classic focal segmental glomerulosclerosis. Am J Kidney Dis 32:72-9

5. Roberti I, Vyas S (2010) Long-term outcome of children with steroid-resistant nephrotic syndrome treated with tacrolimus. Pediatr Nephrol 25:1117-24

6. Sesso R, Santos AP, Nishida SK, Klag MJ, Carvalhaes JT, Ajzen H, Ramos OL, Pereira AB (1992) Prediction of steroid responsiveness in the idiopathic nephrotic syndrome using urinary retinol-binding protein and beta-2-microglobulin. Ann Intern Med 116:905-9

7. Portman RJ, Kissane JM, Robson AM (1986) Use of beta 2 microglobulin to diagnose tubulo-interstitial renal lesions in children. Kidney Int 30:91-8

8. Calişkan S, Hacibekiroğlu M, Sever L, Ozbay G, Arisoy N (1996) Urinary $\mathrm{N}$-acetyl-beta-D-glucosaminidase and beta 2microglobulin excretion in primary nephrotic children. Nephron $74: 401-4$ 
9. Schwartz GJ, Muñoz A, Schneider MF, Mak RH, Kaskel F, Warady BA, Furth SL (2009) New equations to estimate GFR in children with CKD. J Am Soc Nephrol 20:629-637

10. Gao A, Cachat F, Faouzi M, Bardy D, Mosig D, Meyrat BJ, Girardin E, Chehade H (2012) Comparison of the glomerular filtration rate in children by the new revised Schwartz formula and a new generalized formula. Kidney Int 83:524-530

11. DeLong ER, DeLong DM, Clarke-Pearson DL (1988) Comparing the areas under two or more correlated receiver operating characteristic curves: a nonparametric approach. Biometrics 44:837-845

12. Youden WJ (1950) Index of rating diagnostic tests. Cancer 3:32-35

13. Vallés P, Peralta M, Carrizo L, Martin L, Principi I, Gonzalez A, Manucha W (2000) Follow-up of steroid-resistant nephrotic syndrome: tubular proteinuria and enzymuria. Pediatr Nephrol 15:252-8

14. Woroniecki RP, Orlova TN, Mendelev N, Shatat IF, Hailpern SM, Kaskel FJ, Goligorsky MS, O’Riordan E (2006) Urinary proteome of steroid-sensitive and steroid-resistant idiopathic nephrotic syndrome of childhood. Am J Nephrol 26:258-67

15. Woroniecki RP, Shatat IF, Supe K, Du Z, Kaskel FJ (2008) Urinary cytokines and steroid responsiveness in idiopathic nephrotic syndrome of childhood. Am J Nephrol 28:83-90

16. Bolignano D, Donato V, Coppolino G, Campo S, Buemi A, Lacquaniti A, Buemi M (2008) Neutrophil gelatinase-associated lipocalin (NGAL) as a marker of kidney damage. Am J Kidney Dis 52:595-605
17. Nejat M, Hill JV, Pickering JW, Edelstein CL, Devarajan P, Endre ZH (2012) Albuminuria increases cystatin C excretion: Implications for urinary biomarkers. Nephrol Dial Transplant 0:1-8

18. Thielemans N, Lauwerys R, Bernard A (1994) Competition between albumin and low-molecular-weight proteins for renal tubular uptake in experimental nephropathies. Nephron 66:453-458

19. Bernard A, Viau C, Ouled A, Lauwerys R (1987) Competition between low- and high-molecular-weight proteins for renal tubular uptake. Nephron 45:115-118

20. Bernard A, Amor AO, Viau C, Lauwerys R (1988) The renal uptake of proteins: a non selective process in conscious rats. Kidney Int $34: 175-185$

21. Kaseda R, Iino N, Hosojima M, Takeda T, Hosaka K, Kobayashi A, Yamamoto K, Suzuki A, Kasai A, Suzuki Y, Gejyo F, Saito A (2007) Megalin-mediated endocytosis of cystatin C in proximal tubule cells. Biochem Biophys Res Commun 357:1130-1134

22. Stickler GB, Hayles AB, Power MH, Ulrich JA (1960) Renal tubular dysfunction complicating the nephrotic syndrome. Pediatrics 26:75-85

23. Cameron JS (1990) Proteinuria and progression in human glomerular diseases. Am J Nephrol 10:81-7

24. Wasilewska A, Zoch-Zwierz W, Taranta-Janusz K, MichalukSkutnik J (2010) Neutrophil gelatinase-associated lipocalin (NGAL): a new marker of cyclosporine nephrotoxicity? Pediatr Nephrol 25:889-97 